\title{
Erratum to the paper "Reaction Mechanisms and Kinetic Constants used in Mechanistic Models of Coagulation and Fibrinolysis"
}

\author{
(Math. Model. Nat. Phenom., 11 (2016), no. 6, 71-90 \\ DOI: $10.1051 / \mathrm{mmnp} / \mathbf{2 0 1 6 1 1 6 0 5 )}$
}

M. Susree, M. Anand *

Department of Chemical Engineering, Indian Institute of Technology Hyderabad, Kandi, Sangareddy 502285, Telangana, INDIA.

\begin{abstract}
Computational modeling has gained a strong foothold as a tool for understanding and simulating biological phenomena like blood coagulation. However, exceptional complexity of these phenomena (that include hundreds of individual biochemical processes and a few biophysical processes) complicates development of reliable models and interpretation of the results. It is thus of great importance that a model of hemostasis designed for a particular application should be reproducible, apart from making robust predictions. Such a model would further reflect useful pharmacological implications not only with respect to identifying drug targets but also in understanding drug effects on their respective targets. We show, using a sample model, that model predictions vary significantly with the use of different values available in the literature for the same kinetic constant. We thus highlight the importance of having consensus of kinetic constants used in the formulation of mechanistic models for coagulation, and document values for each kinetic constant that can be used in such models, and for varying reaction conditions (synthetic, in vitro, in vivo).
\end{abstract}

Key words: computer simulation, consensus, hemostasis, kinetics, mathematical model

Mathematics Subject Classification: 80A30, 92C45

\section{Correction}

The names of the authors should be M. Susree and M. Anand instead of A.M. Susree and B.M. Anand in the original version of the paper.

${ }^{*}$ Corresponding author. E-mail: anandm@iith.ac.in 